\title{
Plasminogenemia asociada a conjuntivitis leñosa
}

\section{Plasminogen Deficiency and Ligneous Conjunctivitis}

\author{
Melissa de Castro Hernández ${ }^{a}$ \\ Pontificia Universidad Javeriana, Colombia \\ Angélica Natalia Álvarez Guevara ${ }^{b}$ \\ Pontificia Universidad Javeriana, Colombia \\ Iliana De los Reyes Valencia ${ }^{c}$ \\ Pontificia Universidad Javeriana, Colombia
}

a Departamento de Pediatría del Hospital Universitario San Ignacio-Pontificia Universidad Javeriana, Bogotá, Colombia.

b Departamento de Pediatría del Hospital Universitario San Ignacio-Pontificia Universidad Javeriana, Bogotá, Colombia.

c Departamento de Hematooncología Pediátrica del Hospital Universitario San Ignacio-Centro Javeriano de Oncología-Pontificia Universidad Javeriana, Bogotá, Colombia.

Conflictos de interés: declaramos que no tenemos conflictos de interés o sesgo en la publicación de este artículo.

Financiación: no se recibió apoyo por parte de la industria farmacéutica u otras instituciones para la realización del artículo en mención.

Cómo citar: De Castro Hernández M, Álvarez Guevara AN, De Los Reyes Valencia I. Plasminogenemia asociada a conjuntivitis leñosa. Univ. Med. 2018;59(3). doi: https:// doi.org/10.11144/Javeriana.umed59-3.plasm

\section{RESUMEN}

El artículo presenta el caso clínico de un joven de 18 años de edad sin antecedente familiares de enfermedad crónica o hereditaria, nacido a término, sin complicaciones, quien desde los dos días de vida presentó una lesión tipo masa en la conjuntiva tarsal. Inicialmente, recibió tratamiento farmacológico tópico y seguimiento por oftalmología, por conjuntivitis bilateral persistente, masas tarsales recidivantes y cataratas en ambos ojos, por lo que requirió siete intervenciones con pobre respuesta al manejo farmacológico y quirúrgico. A los seis meses de vida se le diagnosticó hidrocefalia, que requirió manejo con derivación del ventrículo peritoneal. Dada la persistencia de la sintomatología y la refractariedad al tratamiento, se ampliaron los estudios y en una junta médica se sugirió el diagnóstico de conjuntivitis leñosa asociada a alteración del plasminógeno. Este diagnóstico fue confirmado por laboratorio clínico, que mostró sus bajas concentraciones de plasminógeno en muestras tomadas con intervalos de dos meses en tres ocasiones: $16,9 \%, 11,1 \%, 18,6 \%$ (valores de referencia: $70-150 \%$ ). Se le indicó heparina de bajo peso molecular antes de procedimientos quirúrgicos mayores y triamcinolona tópica según síntomas oculares.

Palabras clave

Plasminógeno; deficiencia de plasminógeno; fibrina; conjuntivitis leñosa.

\begin{abstract}
We present a case of an 18-year-old patient without a family history of ocular disease, born full term without complications, within his first 2 days a mass in the tarsal conjunctiva appeared. Initially he received topical treatment and follow-up by the ophthalmology department with a diagnosis of persistent bilateral conjunctivitis, relapsing tarsal masses and cataracts in both eyes requiring a total of 7 surgical interventions with a poor response. At the age of 6 months he was diagnosed with hydrocephalus and required a ventricular-peritoneal shunt. Given the persistence of the symptoms, further studies were made and a medical board made the diagnosis of ligneous conjunctivitis associated to low levels of plasminogen. The diagnosis was confirmed by decreased levels of plasminogen in serum measured three times with 2 months intervals: $16.9 \%, 11.1 \%, 18.6 \%$ (reference values $70-150 \%$ ). Low molecular weight
\end{abstract}


heparin was ordered before surgical procedures, and topical triamcinolone applied according to ocular symptoms.

Keywords

Plasminogen; plasminogen deficiency; ligneous conjunctivitis.

\section{Introducción}

La fibrinólisis es el proceso mediante el cual la fibrina innecesaria es eliminada del árbol vascular; en otras palabras, es un sistema reactivo a la activación de la coagulación y a la generación final de trombina. Así, la plasmina, en condiciones normales, circula en el plasma en forma de proenzima: llamada plasminógeno. Al ser la transformación del plasminógeno en plasmina un proceso determinado por la presencia de activadores del plasminógeno, conocidos como activador tisular del plasminógeno y activador del plasminógeno tipo urocinasa.

Los principales sistemas implicados en la regulación de las tenazas de la coagulación y formación de coágulos de fibrina son cuatro: la proteína del sistema C, la antitrombina, el factor tisular inhibidor y el sistema fibrinolítico.

El sistema fibrinolítico es necesario para la disolución y destrucción de coágulos. Dos componentes principales del sistema fibrinolítico son el plasminógeno y el activador tisular del plasminógeno (1).

El plasminógeno, también llamado profibrinolisina, es una glicoproteína de $92 \mathrm{kd}$, sintetizada por el hígado, que es el precursor inactivo de la plasmina. Este plasminógeno es codificado por un gen que se encuentra en el cromosoma 6 que posee 791 aminoácidos y 5 estructuras homólogas denominadas kringles que le confieren gran afinidad por la fibrina, alfa2 antiplasmina y la trombospondina $(2,3)$.

La vida media del plasminógeno en personas sanas es de aproximadamente 2 a 3 días, siendo más corta en pacientes con hipoplasminogenemias congénitas y algunos tipos de displasminogenemias, enfermedades que se han asociado con problemas trombóticos a repetición a nivel intrafamiliar; sin embargo, no se consideran una causa muy prevalente de trombofilia (1).
El objetivo de este reporte de caso es conocer el comportamiento de esta rara enfermedad, sus características clínicas y cuándo sospecharla.

\section{Reporte de caso}

El caso corresponde a un joven de 18 años de edad, remitido por el servicio de oftalmología al servicio de hematooncología pediátrica a los 12 años de edad por sospecha de conjuntivitis leñosa: "persistencia de masa tarsal con respuesta fallida a la terapia, lesiones orales e hidrocefalia". Es el tercer hijo de la unión, sin antecedente de enfermedad ocular familiar. Como único dato positivo y relevante durante controles prenatales, la madre presentó un cuadro de varicela en la semana 25 de gestación. Nació a término, de 38 semanas sin complicaciones.

A los dos días de vida presentó en los párpados una lesión tipo masa que requirió hospitalización y manejo farmacológico tópico con pobre repuesta. Se le documentó hidrocefalia a los seis meses de vida que requirió un catéter de derivación ventrículo-peritoneal. La hidrocefalia se le atribuyó como posible etiología el antecedente de la varicela gestacional. Continuó manejo interdisciplinario por el servicio de neurología pediátrica sin signos agudos de lesión neurológica, pero con limitación para el aprendizaje, además del seguimiento por el servicio de oftalmología, dado el deterioro de la agudeza visual y persistencia de secreción verdosa conjuntival refractaria a múltiples tratamientos. (figuras 1 y 2 ). 


\section{Figura 1}

Secreción verdosa persistente. Corresponde a nuestro paciente.

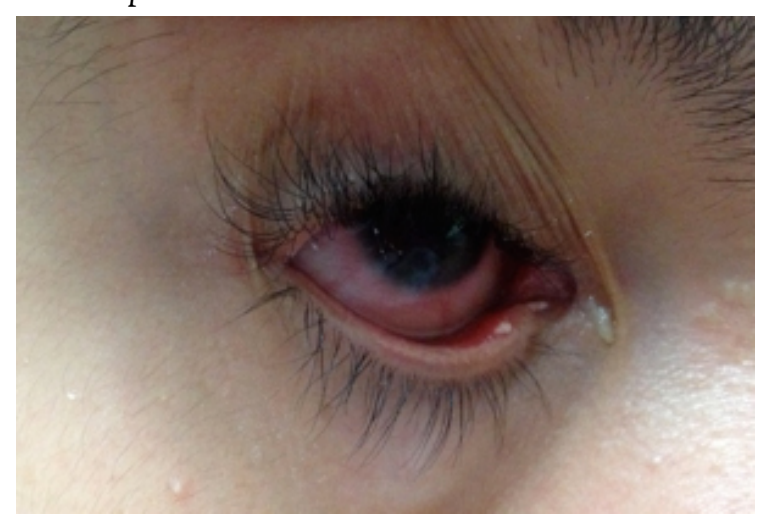

Autorización de la madre y el paciente para publicación de fotos

\section{Figura 2}

Seguimiento del paciente. Corresponde a nuestro paciente.

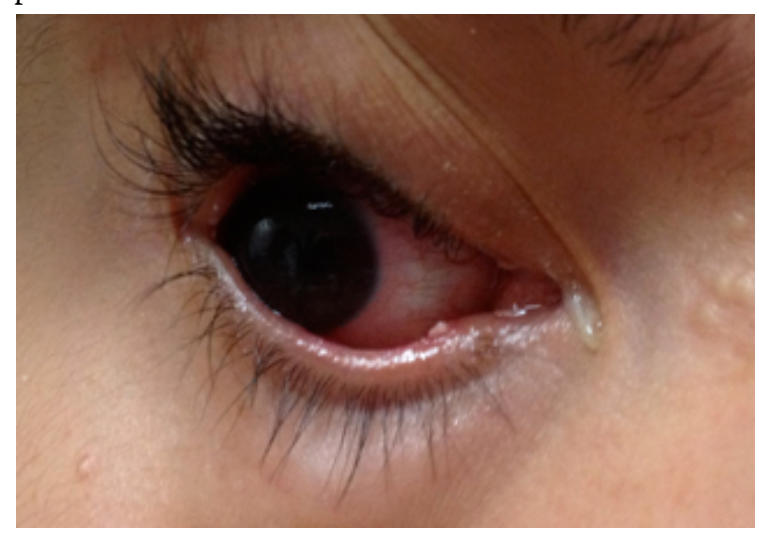

Autorización de la madre y el paciente para publicación de fotos

En total, el joven requirió siete cirugías (entre ellas la de catarata documentada a los 4 años e intervenida con cirugía a los 12 años). La última cirugía fue a los 16 años de edad por causa de una masa tarsal recidivante (figura 3) con hallazgos anatomopatológicos inconclusos.

\section{Figura 3}

Lesiones oculares tarsales. Corresponde a nuestro paciente.

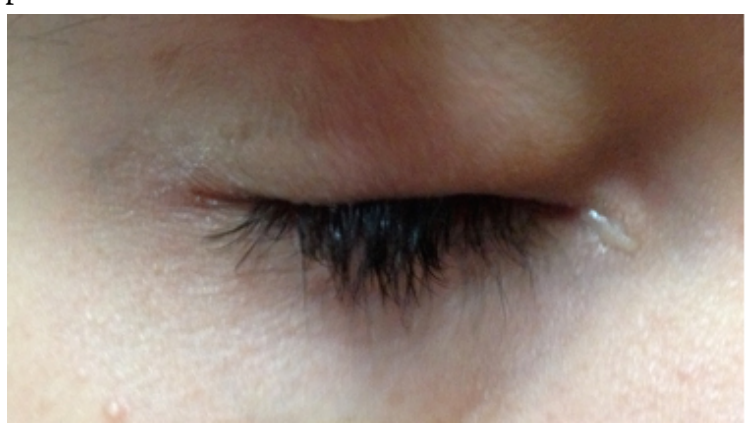

Autorización de la madre y el paciente para publicación de fotos

A los 12 años, teniendo en cuenta la persistencia de la sintomatología y los hallazgos clínicos de masa tarsal recurrente, hidrocefalia obstructiva se consideró en una junta médica un cuadro compatible con conjuntivitis leñosa. Este diagnóstico fue confirmado por laboratorio clínico, que evidenció bajas concentraciones de plasminógeno, muestras tomadas con intervalos de dos meses en tres ocasiones en laboratorio de referencia para hemostasia: 16,9\%, 11,1\% y $18,6 \%$ (valores de referencia: $70-150 \%$ ). Se indicó manejo con heparina de bajo peso molecular antes de cualquier procedimiento quirúrgico mayor, y triancinolona tópica, tratamiento que disminuyó la aparición de las lesiones oculares. Al paciente, durante su manejo integral y multidisciplinario, se le diagnosticó epilepsia a los 15 años de edad, en manejo con levetircetam sin nuevas crisis. Cursa séptimo grado de bachillerato en una institución para personas con capacidades funcionales diferentes, y aunque la mayoría de sus actividades las hace solo, requiere acompañamiento para desplazarse por la alteración de su agudeza visual, secundaria a la patología de base.

\section{Discusión}

El plasminógeno se encuentra presente en el plasma y la mayor parte en el líquido extracelular como precursor inactivo de una enzima proteasa llamada plasmina. Es el componente central del 
sistema fibrinolítico e interviene en la disolución de los coágulos. Su concentración normal en sangre está entre 1,5 y $2 \mu \mathrm{mol} / \mathrm{l}$ o $20 \mathrm{mg} / \mathrm{dl}$.

La activación del plasminógeno por el activador tisular del plasminógeno es la principal vía que conduce a lisis de los coágulos de fibrina; mientras que la activación del plasminógeno por el activador del plasminógeno tipo urocinasa es responsable de mediar la activación del plasminógeno en asociación con la superficie de las células y parece iniciar la mayor parte de las actividades no fibrinolíticas de la plasmina.

Una deficiencia del plasminógeno o del activador tisular del plasminógeno reduce la capacidad de eliminar la formación excesiva de coágulos y contribuir a la enfermedad tromboembólica.

La prevalencia de este trastorno se desconoce. Dada la baja prevalencia de la enfermedad, en Escocia se realizó una evaluación de más de 9000 donantes de sangre en y reveló una prevalencia de 2,9 casos por cada 1000 sujetos heterocigotos, los cuales tenían deficiencias cuantitativas asintomáticas del plasminógeno, siendo la más frecuente la tipo I. Con estos datos, la prevalencia teórica y relativa de homocigotos o heterocigotos compuestos se ha calculado de 1,6 por cada 1.000 .000 nacidos vivos $(2,3,4,5,6)$; sin embargo, se necesitan más estudios epidemiológicos y evidencia que soporten esta estadística.

Los casos de pacientes con deficiencia tipo I cuantitativa del plasminógeno han sido reportados en todo el mundo. Parece haber un mayor número de casos identificados e informados de Turquía o en pacientes de ascendencia turca, tal vez relacionado con el efecto de los matrimonios mixtos en algunas comunidades.

La deficiencia del plasminógeno tipo II cualitativo parece ser más común en otras poblaciones. Un estudio en Japón demostró una prevalencia de heterocigotos del 3,83\%; otros realizados en China y Corea notificaron una prevalencia del $1,5 \%$ y del $1,6 \%$, respectivamente $(3,5,6)$.

La deficiencia del plasminógeno es una enfermedad rara y cursa con manifestaciones clínicas múltiples y variables que no se relacionan con los valores o niveles séricos encontrados. La deficiencia de plasminógeno se clasifica con base en el patrón de trasmisión en hereditaria o adquirida; La coagulación intravascular diseminada es el máximo representante de esta condición $(2,3)$. También se ha documentado un patrón de herencia autosómico recesivo asociado a conjuntivitis leñosa e hidrocefalia obstructiva. Existe otra clasificación basada en el compromiso numérico y función: cuantitativa o tipo I (hipoplasminogenemia: con valores de plasminógeno entre $4 \%$ y $51 \%$ ) y la cualitativa o tipo II (displasminogenemia).

Esta patología puede afectar diversos órganos. La enfermedad suele tener como manifestación inicial la conjuntivitis leñosa, denominada asî por los hallazgos de fibrina en la biopsia como componente predominante en las membranas, así como la presencia de depósitos subepiteliales de fibrina intacta. Estas manifestaciones fueron descritas en los pacientes con deficiencia de plasminógeno tipo I y no aparecen en los pacientes con diagnóstico de aplasminogenemia tipo II. Este tipo de alteración conjuntival suele ser bilateral, recurrente y persistir durante años. Puede aparecer en cualquier edad de la vida, aunque se han reportado mayoría de casos en niños y género femenino.

Los hallazgos histológicos de la conjuntivitis leñosa describen un material, eosinofílico, amorfo, que sustituye casi todo el tejido conectivo, con pocos vasos sanguíneos e inflamación ligera constituida por linfocitos y escasos polimorfonucleares neutrófilos. Esta alteración crónica de acumulación de membranas forman masas hipertróficas de color blanco rosado que terminan por alterar y sustituir la conjuntiva tarsal superior e inferior; en su evolución estas masas dan lugar a sensación de cuerpo extraño con lagrimeo, inyección conjuntival crónica (sobre todo alrededor del tejido anómalo) y ciliar con afectación de la córnea $(7,8)$.

En la piel y mucosas presentan alteración de la fibrinólisis y acumulo de fibrina a nivel subepitelial, lo cual conduce a una cicatrización aberrante que puede afectar distintas mucosas 
del organismo como son: la cavidad oral, la nasofaringe, la tráquea y la vagina.

En el cerebro es interesante anotar también la presencia de casos de hidrocefalia oclusiva. Esta patología tiene un curso crónico y recurrente; además, se han reportado rangos de evolución de la enfermedad desde 4 meses hasta 44 años y, en ocasiones, hay resolución espontánea $(7,8,9)$.

Para confirmar este trastorno se deben realizar pruebas que evalúen la actividad del plasminógeno y pruebas de antígenos. Debido a que las lesiones leñosas son más frecuentes en la deficiencia del plasminógeno tipo I, la prueba sola de antígeno es insuficiente para descartar esta entidad, pues hay algunos estudios que han reportado valores normales del antígeno en los pacientes y miembros de su familia, lo que sugiere un defecto funcional en la proteína. Para diagnosticar la conjuntivitis leñosa secundaria a la deficiencia de plasminógeno se requieren hallazgos clínicos y paraclínicos.

Las lesiones membranosas o leñosas deben ser documentadas con los cambios histológicos clásicos. La historia familiar puede ayudar a apoyar el diagnóstico si otros hermanos afectados o familiares tienen el mismo padecimiento (5).

Se han evaluado numerosas estrategias de manejo con una pobre y variable respuesta desde la extirpación quirúrgica hasta manejo local con heparina, corticoide, ciclosporina, azatioprina, hialuronidasa y quimotripsina (9). Se evidencia mejoría de las lesiones de la conjuntiva con el uso de altas dosis de corticoide; sin embargo, la única terapia eficaz documentada en el momento que conduce a la resolución y evita la regeneración de las lesiones es el uso tópico o sistémico de concentrados de plasminógeno, aun cuando estos no están disponibles. Además, es importante anotar que la deficiencia de plasminógeno cursa con manifestaciones sistémicas y cada una de ellas requiere un tratamiento específico; por lo tanto, se hace indispensable un manejo interdisciplinario.

Existen otras formas de terapias farmacológicas solas o combinadas con cirugía para eliminar las seudomembanas, como los agentes trombolíticos, que contienen plasminógeno y soluciones oftálmicas a base de anistreplasa (combinación de estreptoquinasa bacteriana y plasminógeno), la cual mostró mayor efectividad con su uso prolongado después de la cirugía.

El pronóstico de esta enfermedad es variable en función de la magnitud, duración y lugar de los síntomas, y aunque muchos pacientes identificados han vivido hasta la edad adulta, algunos de ellos han muerto a causa de los efectos de este trastorno.

La calidad de vida de los pacientes sin terapia eficaz es pésima. Suelen presentar comorbilidades asociadas con deterioro de algunos órganos, por ejemplo, pérdida de la visión, lo que limita valerse por sí mismos; o alteraciones de la dentición y de la vía respiratoria, que pueden resultar en falla ventilatoria y muerte (9).

La plasminogenemia asociada a conjuntivitis leñosa e hidrocefalia es una entidad poco frecuente, lo cual limita las posibilidades de realizar estudios clínicos que demuestren la eficacia de algunos tratamientos.

A pesar que la conjuntivitis leñosa se describió por primera vez en 1964, solo hasta 1997 se asoció con el déficit de plasminógeno; por tanto, es importante tener un alto índice de sospecha para hacer este tipo de diagnósticos.

En pacientes que no tengan adecuada respuesta a un tratamiento específico para la patología, se deben hacer diagnósticos diferenciales antes de extender tratamientos fallidos.

\section{Referencias}

1. Angles-Cano E. Structural basis for the pathophysiology of lipoprotein(a) in the athero-thrombotic process. Braz J Med Biol Res. 1997;30(11):1271-80.

2. J Sans-Sabrafen, Besses Raebel C, Vives JL. Hematología clínica. 5a ed. Madrid: Elsevier; 2006.

3. Mehta R, Shapiro AD. Plasminogen deficiency. Haemophilia. 2008;14(6):1261-8.

4. Orkin SH, Nathan DG, Ginsburg D, Look AT, Fisher DE, Lux IV 
S. Nathan and Oski's hematology of infancy and childhood. Philadelphia: Elsevier; 2009.

5. Schuster V, Mingers AM, Seidenspinner S, Nüssgens Z, Pukrop T, Kreth HW. Homozygous mutations in the plasminogen gene of two unrelated girls with ligneous conjunctivitis. Blood. 1997;90(3):958-66.

6. Furlan AJ, Lucas FV, Craciun R, Wohl RC. Stroke in a young adult with familial plasminogen disorder. Stroke. 1991;22(12):1598-602.

7. Emonts M, Wiertsema SP, Veenhoven RH, Houwing-Duistermaat JJ, Walraven $\mathrm{V}$, de Groot $\mathrm{R}$, et al. The $4 \mathrm{G} / 4 \mathrm{G}$ plasminogen activator inhibitor-1 genotype is associated with frequent recurrence of acute otitis media. Pediatrics. 2007;120(2):e317-23.

8. Ozçelik U, Akçören Z, Anadol D, Kiper N, Orhon M, Göçmen A, et al. Pulmonary involvement in a child with ligneous conjunctivitis and homozygous type I plasminogen deficiency. Pediatr Pulmonol. 2001;32 (2):179-83.

9. Pergantou H, Likaki D, Fotopoulou M, Katsarou O, Xafaki P, Platokouki H. Management of ligneous conjunctivitis in a child with plasminogen deficiency. Eur J Pediatr. 2011;170(10):1333-6. 